\title{
From traditional navigation maps to future high-definition life maps for automated vehicles
}

Ralf G. Herrtwich

HERE Technologies

This manuscript is not available according to publishing restriction. Thank you for your understanding. 